TITLE:

\title{
Molecular orientation dynamics on the structural rheology in diblock copolymers
}

$\operatorname{AUTHOR}(S)$ :

Yabunaka, Shunsuke; Ohta, Takao

CITATION:

Yabunaka, Shunsuke ...[et al]. Molecular orientation dynamics on the structural rheology in diblock copolymers. Soft Matter 2013, 9(31): 74797488

ISSUE DATE:

2013

URL:

http://hdl.handle.net/2433/190415

\section{RIGHT:}

This journal is @ The Royal Society of Chemistry 2013; この論文は出版 社版でありません。引用の際には出版社版をご確認ご利用ください。; This is not the published version. Please cite only the published version. 


\title{
Molecular orientation dynamics on the structural rheology in diblock copolymers
}

\author{
Shunsuke Yabunaka and Takao Ohta \\ Received Xth XXXXXXXXXX 20XX, Accepted Xth XXXXXXXXX 20XX \\ First published on the web Xth $X X X X X X X X X X 200 X$ \\ DOI: 10.1039/b000000x
}

We formulate a viscoelastic theory of micro-phase separated structures in diblock copolymers in which the local composition is coupled with the molecular orientation. The linear viscoelastic response is investigated for aligned lamellar structures. Three kinds of complex moduli and viscosities characterizing an incompressible lamellar structure are obtained up to the leading order of the coupling.

\section{Introduction}

Diblock copolymers exhibit various periodic microphase structures in thermal equilibrium ${ }^{1-11}$. Rheology of these mesoscopic structures has been investigated to characterize their dynamical properties. See, for instance, ref. ${ }^{12}$ and the earlier references therein. It is mentioned that different phases show different rheological properties and hence rheological measurements are useful to obtain the evidence of the morphological transitions ${ }^{13-16}$.

There are many computational and analytical studies on the structural rheology of microphase separated diblock copolymers. Yoo and Vinals ${ }^{17}$ considered the effects of anisotropic diffusion and hydrodynamic interaction. They found that anisotropy of diffusion influences the grain boundary motion significantly. Giupponi et al ${ }^{18}$ studied gyroid structure in flow by computer simulations of amphiphillic fluids by means of the lattice Boltzmann method. They found fluid-like behavior for low frequency of viscoelastic response under an oscillatory shear flow. Tamate et al ${ }^{19}$ formulated an analytical theory of viscoelasticity for interconnected double gyroid structures. They considered concentration variation under shear flow and calculated the energy dissipation due to the deformation of interconnected structures in the weak segregation limit. The reversible part of the stress tensor which arises from the concentration variations was calculated by the mode expansion. Their theory relies on the fact that, in the case of a double gyroid structure, there are two different magnitudes of reciprocal lattice vectors ${ }^{22}$. When the periodic structure is represented by the fundamental reciprocal lattice vectors having only one magnitude, such as lamellar, hexagonal and body-centeredcubic (BCC) structures, the linear part of loss modulus is absent $^{19}$.

Department of Physics, Kyoto University, Kyoto 606-8502, Japan. ; E-mail: yabunaka@scphys.kyoto-u.ac.jp
The above fact implies that one needs generally to introduce other kinetic variables to describe the structural rheology of phase-separated diblock copolymers. This is also required by another consideration. In an aligned lamellar structure in an incompressible system, there are generally three kinds of viscosities ${ }^{20}$. However, it is readily shown by symmetry consideration that those viscosities cannot be obtained by a formulation which contains only a scalar variable such as a local concentration. Therefore, although the theories in terms of local concentration have successfully predicted the equilibrium phase diagram for micro-phase separated states ${ }^{1,6-8}$, vector or tensor variables are necessary to describe the dynamics, in particular, rheology of mesoscopic periodic structures. We will return to this problem in section 5 .

In the present paper, we address this problem. To the authors' knowledge, no theories have been available so far to derive three kinds of viscosities or three kinds of complex moduli for lamellar structure in diblock copolymer melts. It is well known that diblock copolymer molecules are elongated in a phase-separated state in the direction parallel to the compositional gradient ${ }^{21}$. This occurs even for flexible chains. Therefore we may introduce a local orientational order coupled to the local concentration. These are perturbed differently by an external flow and influences the rheological response of the system. We construct dynamic equations for the compositional field and the orientational order parameter field. We analyze these equations by means of the mode expansion in the weak segregation limit and obtain the rheological response of this system. This approach is related, to some extent, with the theory of smectic liquid crystals. See for example ref. ${ }^{23}$. In this formulation, a phase variable to represent the one-dimensional periodic structure is coupled to the orientational degree of freedom of molecules where, however, complex compliances are not concerned.

Lamellar structure under shear flow becomes unstable since 
the period tends to be different from the equilibrium one ${ }^{24}$. There are theoretical studies for both block copolymers ${ }^{25,26}$ and smectic liquid crystals $23,27-29$. This nonlinear effect is not considered in the present paper but we are restricted to the linear response focusing our attention on the relations between several kinds of frequency-dependent viscosities.

The organization of the present paper is as follows. In the next section (section 2), we introduce a kinetic model which consists of the local concentration and the local orientational order parameter. In section 3, general viscoelastic properties are given for lamellar structure under shear flow. The complex moduli are calculated up to the first order of the coupling between the concentration and the orientation degrees of freedom in section 4. Concluding remarks and discussion are given in section 5. In Appendix A, the macroscopic stress tensor is derived in terms of the local concentration and the orientational field whereas, in Appendix B, some of the details of the viscoelastic response are presented. In Appendix $\mathrm{C}$, the details of the procedure is described to solve the timeevolution equations by the perturbation expansion in terms of the coupling strength of the concentration and the orientational field, and the strength of the shear rate.

\section{Model}

We consider A-B type diblock copolymer melts. One of the basic quantities is the local volume fraction of the A block denoted by $\phi(\vec{x})$. The other is the tensor of the orientational order $Q_{i j}(\vec{x})$ defined by

$$
Q_{i j}(\vec{x})=\left\langle m_{i} m_{j}-\frac{1}{3} \delta_{i j}\right\rangle,
$$

where $m_{i}$ is the end-to-end vector for the $i$ th molecule. Note that this tensor is position-dependent since $\langle\cdots\rangle$ means a local average in a finite volume around a position $\vec{x}$, which is much larger than the atomic scale and much smaller than the characteristic length of deformations of phase separated pattern. The simplest expression of the free energy for micro-phase separation in A-B type diblock copolymer is given by

$$
\begin{aligned}
F= & \int d \vec{x}\left[\frac{1}{2} L|\vec{\nabla} \phi(\vec{x})|^{2}-\frac{\theta}{2} \phi(\vec{x})^{2}+\frac{g}{4} \phi(\vec{x})^{4}+G\left(Q_{i j}\right)\right. \\
& \left.-\beta Q_{i j}\left(\nabla_{i} \phi\right)\left(\nabla_{j} \phi\right)+\frac{1}{2} C\left(\nabla_{k} Q_{i j}\right)\left(\nabla_{k} Q_{i j}\right)\right] \\
& +\frac{\alpha}{2} \int d \vec{x} \int d \vec{y} H(\vec{x}-\vec{y})[\phi(\vec{x})-\bar{\phi}][\phi(\vec{y})-\bar{\phi}] .
\end{aligned}
$$

The repeated indices implies summation. The free energy without the orientational order parameter was derived by Ohta and Kawasaki ${ }^{30}$. The constants $L, \theta, g$, and $\alpha$ are all positive in a microphase separated state. $\bar{\phi}$ is the spatial average of $\phi$.
The term $-\beta Q_{i j}\left(\nabla_{i} \phi\right)\left(\nabla_{j} \phi\right)$ represents the coupling between the orientational order parameter and the concentration field. When $\beta>0$ as we assume, each diblock copolymer molecule tends to align in the direction of the composition gradient. The homogeneous part of the free energy $G\left(Q_{i j}\right)$ for the orientational field takes the simplest form

$$
G\left(Q_{i j}\right)=\frac{a}{2} Q_{i j} Q_{i j}
$$

where $a>0$ assuming that molecules do not cause spontaneous alignment. The kernel $H(\vec{x})$ in the last term in eqn (2) is defined by

$$
-\nabla^{2} H(\vec{x}-\vec{y})=\delta(\vec{x}-\vec{y}) .
$$

This Coulomb-type long range interaction originates from the osmotic incompressibility of diblock copolymer melts ${ }^{30}$ or, in other words, from the correlation-hole effects ${ }^{31}$.

In order to derive the time-evolution equations, we employ the variational method developed by Doi for dynamics of various soft matter ${ }^{32}$. We introduce the local velocity of A and B components as $\vec{v}_{A}$ and $\vec{v}_{B}$, respectively and define the volume average velocity as

$$
\vec{v}=\phi \vec{v}_{A}+(1-\phi) \vec{v}_{B}
$$

and the relative velocity between the two components as

$$
\vec{w}=\vec{v}_{A}-\vec{v}_{B}
$$

The time evolution equation for the local volume fraction obeys the continuity equation

$$
\frac{\partial \phi}{\partial t}+\vec{\nabla} \cdot\left(\vec{v}_{A} \phi\right)=0 .
$$

We employ the variational method to derive the time-evolution equations. The variational functional is introduced in the present problem as

$$
R=\Psi+\dot{F}-\int d \vec{x}\left(p(\vec{x}) \vec{\nabla} \cdot \vec{v}+q \dot{Q}_{i i}\right),
$$

where $p$ is determined by the incompressibility condition $\vec{\nabla} \cdot \vec{v}=0$, and the Lagrange multiplier $q$ is determined by the traceless condition $Q_{i i}=0$. The dissipation function $\Psi$ is defined by

$$
\begin{array}{ll} 
& \Psi=\int d \vec{x}\left[\frac{1}{2} v_{i j k l} A_{i j} A_{k l}+\frac{\zeta_{i j}}{2}\left(v_{A}-v\right)_{i}\left(v_{A}-v\right)_{j}\right. \\
+\quad & \left(\dot{Q}_{i j}+v_{m}\left(\nabla_{m} Q_{i j}\right)+Q_{j m} \Omega_{m i}+Q_{i m} \Omega_{m j}-\lambda_{i j m n} A_{m n}\right) \\
\times \quad & \left(\dot{Q}_{k l}+v_{n}\left(\nabla_{n} Q_{k l}\right)+Q_{l n} \Omega_{n k}+Q_{k n} \Omega_{n l}-\lambda_{k l m n} A_{m n}\right) \\
\times \quad & \left.\frac{1}{2} \tilde{\Gamma}_{i j k l}\right],
\end{array}
$$


where $\zeta_{i j}, \tilde{\Gamma}_{i j k l}$ and $\lambda_{i j k l}$ are constants. We assume that $v_{i j k l}$ depends on $Q_{i j}$ as eqn (21) below. The symmetric velocity gradient tensor $A_{i j}$ is defined as

$$
A_{i j}=\frac{1}{2}\left(\frac{\partial v_{i}}{\partial x_{j}}+\frac{\partial v_{j}}{\partial x_{i}}\right)
$$

and the antisymmetric one $\Omega_{i j}$ as

$$
\Omega_{i j}=\frac{1}{2}\left(\frac{\partial v_{i}}{\partial x_{j}}-\frac{\partial v_{j}}{\partial x_{i}}\right) .
$$

The variations $\delta R / \delta \vec{v}_{A}=0, \delta R / \delta \vec{v}=0$ and $\delta R / \delta \dot{Q}_{i j}=0$ together with eqn (7) give us

$$
\begin{gathered}
\frac{\partial \phi}{\partial t}+\vec{\nabla} \cdot(\vec{v} \phi)=\nabla_{i}\left[D_{i j} \nabla_{j} \frac{\delta F}{\delta \phi}\right] \\
\dot{Q}_{i j}+\nabla_{n}\left(v_{n} Q_{i j}\right)+Q_{i k} \Omega_{k j}+Q_{j k} \Omega_{k i}-\lambda_{i j k l} A_{k l} \\
=\quad-\Gamma_{i j k l}\left(\psi_{k l}-\frac{1}{3} \delta_{k l} \psi_{m m}\right), \\
-\quad \nabla_{j}\left(v_{i j k l} A_{k l}+\lambda_{k l i j} \psi_{k l}+\psi_{i k} Q_{k j}-\psi_{j k} Q_{k i}-p \delta_{i j}\right) \\
+\phi \nabla_{i} \frac{\delta F}{\delta \phi}-\left(\nabla_{i} Q_{k l}\right) \psi_{k l}=0,
\end{gathered}
$$

where $D_{i j}=\phi\left(\zeta^{-1}\right)_{i j}$ and

$$
\begin{gathered}
\tilde{\Gamma}_{i j m n} \Gamma_{m n k l}=\frac{1}{2}\left(\delta_{i k} \delta_{j l}+\delta_{i l} \delta_{j k}-\frac{2}{3} \delta_{i j} \delta_{k l}\right), \\
\psi_{i j}=\frac{\delta F}{\delta Q_{i j}}=a Q_{i j}-C \nabla^{2} Q_{i j}-\beta \nabla_{i} \phi \nabla_{j} \phi .
\end{gathered}
$$

In eqn (13), the term $Q_{i k} \Omega_{k j}+Q_{j k} \Omega_{k i}$ represents the rotation of the molecular orientation in the rotational flow and the term $-\lambda_{i j k l} A_{k l}$ represents alignment of molecules in the flow. We have assumed that the relative velocity field $\vec{w}$ does not couple with the time evolution of the orientational field. This is justified when the dynamical properties of the two components, such as the friction constant, are same in the two blocks.

The coefficients $v_{i j k l}$ and $\lambda_{i j k l}$ have the following properties. First of all, we can assume that $v_{i j k l}$ satisfies

$$
\begin{aligned}
& v_{i j k l}=v_{j i k l}=v_{i j l k}=v_{j i l k}, \\
& v_{i i k l}=v_{i j k k}=0,
\end{aligned}
$$

which follows from $A_{i j}=A_{j i}$ and $A_{i i}=0$. Onsager's reciprocity is represented as

$$
v_{i j k l}=v_{k l i j} .
$$

Similarly we may put

$$
\lambda_{i j k l}=\lambda_{j i k l}=\lambda_{i j l k}=\lambda_{j i l k},
$$

$$
\lambda_{i i k l}=0, \lambda_{i j k k}=0,
$$

which follows from $A_{i j}=A_{j i}, Q_{i j}=Q_{j i}$ and $A_{i i}=Q_{i i}=0$. We expand $v_{i j k l}$ in terms of $Q_{i j}$ as ${ }^{33}$

$$
\begin{aligned}
v_{i j k l}= & v_{1}\left(\delta_{i k} \delta_{j l}+\delta_{j k} \delta_{i l}-\frac{2}{3} \delta_{i j} \delta_{k l}\right) \\
& +v_{2}\left(\delta_{i k} Q_{j l}+\delta_{j k} Q_{i l}+\delta_{j l} Q_{i k}+\delta_{i l} Q_{j k}\right. \\
& \left.-\frac{4}{3} \delta_{i j} Q_{k l}-\frac{4}{3} \delta_{k l} Q_{i j}\right)+O\left(Q_{i j}^{2}\right) .
\end{aligned}
$$

It will be shown in Appendix $C$ that this expansion turns out to be an expansion in terms of the coupling strength $\beta$.

Hereafter we set the coefficients $\lambda_{i j k l}, \Gamma_{i j k l}, D_{i j}$ to be isotropic constants as $\lambda \delta_{i k} \delta_{j l}, \Gamma \delta_{i k} \delta_{j l}, D \delta_{i j}$ although these may be anisotropic generally and functions of $Q_{i j}$ and $\phi$. This can be justified in the weak segregation limit where the composition variation and the orientational order is weak over the space. From the above relations, the time evolution equations (12) and (13) are given, respectively, by

$$
\begin{gathered}
\frac{\partial \phi}{\partial t}+\vec{\nabla} \cdot(\vec{v} \phi)=D \nabla^{2} \frac{\delta F}{\delta \phi}, \\
\dot{Q}_{i j}+\nabla_{n}\left(v_{n} Q_{i j}\right)+Q_{i k} \Omega_{k j}+Q_{j k} \Omega_{k i}-\lambda A_{i j} \\
=-\Gamma\left[a Q_{i j}-C \nabla^{2} Q_{i j}-\beta\left(\left(\nabla_{i} \phi\right)\left(\nabla_{j} \phi\right)\right.\right. \\
\left.\left.-\frac{1}{3} \delta_{i j}\left(\nabla_{k} \phi\right)\left(\nabla_{k} \phi\right)\right)\right],
\end{gathered}
$$

where we assume that $\lambda>0$ such that molecules tend to extend to the flow direction.

The total macroscopic stress tensor consists of two parts

$$
\Sigma_{i j} \equiv \frac{1}{V} \int d \vec{x} \sigma_{i j}=\frac{1}{V} \int d \vec{x} \sigma_{i j}^{r}+\frac{1}{V} \int d \vec{x} \sigma_{i j}^{d},
$$

with $V$ the system volume. The reversible part can be derived from the free energy (2) as

$$
\begin{aligned}
\Sigma_{i j}^{r} & =\frac{1}{V} \int d \vec{x} \sigma_{i j}^{r} \\
& =-\frac{1}{V} \int d \vec{x}\left[L\left(\nabla_{j} \phi(\vec{x})\right)\left(\nabla_{i} \phi(\vec{x})\right)\right. \\
& \left.+C\left(\nabla_{j} Q_{k l}(\vec{x})\right)\left(\nabla_{i} Q_{k l}(\vec{x})\right)\right] \\
& +\frac{\alpha}{2 V} \int d \vec{x} \int d \vec{y}\left[\nabla_{i} H(\vec{x}-\vec{y})\right]\left(x_{j}-y_{j}\right) \phi(\vec{x}) \phi(\vec{y}) \\
& +\frac{\beta}{V} \int d \vec{x}\left[Q_{i k}\left(\nabla_{k} \phi(\vec{x})\right)\left(\nabla_{j} \phi(\vec{x})\right)\right. \\
& \left.+Q_{j k}\left(\nabla_{k} \phi(\vec{x})\right)\left(\nabla_{i} \phi(\vec{x})\right)\right]+\frac{\lambda}{V} \int d \vec{x} \psi_{i j} .
\end{aligned}
$$

When the orientational order parameter is absent, this agrees with that derived by Kawasaki and Ohta ${ }^{34}$. We describe the 
detail of the derivation of (25) in Appendix A. The dissipative part of the stress tensor is given from eqn (14) and (21) by

$$
\begin{aligned}
\Sigma_{i j}^{d} & =\frac{1}{V} \int d \vec{x} \sigma_{i j}^{d}=\frac{1}{V} \int d \vec{x}\left(v_{i j k l} A_{k l}\right) \\
& =2 v_{1} A_{i j}+2 v_{2}\left(Q_{j l} A_{i l}+Q_{i l} A_{j l}-\frac{2}{3} \delta_{i j} Q_{k l} A_{k l}\right) \\
& +O\left(Q_{i j}^{2}\right) .
\end{aligned}
$$

The incompressibility condition $A_{i i}=0$ has been used.

\section{Theory of linear viscoelastic response for lamellar structures}

In this section we investigate the linear viscoelastic response for lamellar structures under an oscillatory shear flow given by

$$
v_{i}\left(x_{i}, t\right)=\dot{\gamma}_{i j}(t) x_{j}
$$

with

$$
\gamma_{i j}(t)=\gamma_{0 i j} \sin \omega t
$$

Here $\gamma_{0 i j}$ is a constant second rank tensor. There might be fluctuations of the local velocity field around the shear flow (27) due to deformations of the microphase separated structure. But this hydrodynamic effects can be neglected in the case of block copolymer melts except for extremely low frequency regime. It has been argued in $\operatorname{ref}^{19}$ that when the melt viscosity is proportional to the molecular weight ${ }^{35}$, the hydrodynamic effects are not important in the weak segregation regime. Actually many viscoelastic theories ignore the hydrodynamic effects. See, e.g., refs ${ }^{36,37}$. Moreover, experimental data for block copolymer melts are found to be fitted by the Rouse model without hydrodynamic effects ${ }^{38}$.

We consider the viscoelastic response of the macroscopic stress tensor (25) for an aligned lamellar structure. Before carrying out detailed calculations, we discuss the general properties. The normal unit vector $\vec{n}$ is defined such that it is parallel to the fundamental reciprocal vector of the lamellar structure in the absence of shear flows. Without loss of generality, we may assume that $\vec{n}$ is parallel to the $x$ axis. Up to linear order of the shear strength, we note that there are three contributions by symmetry argument

$$
\begin{aligned}
\Sigma_{i j} & =\frac{1}{2}\left(\gamma_{0 i j}+\gamma_{0 j i}\right)\left(G_{1}^{\prime}(\omega) \sin \omega t+G_{1}^{\prime \prime}(\omega) \cos \omega t\right) \\
& +\frac{1}{2}\left(\gamma_{0 k l}+\gamma_{0 l k}\right)\left(G_{2}^{\prime}(\omega) \sin \omega t+G_{2}^{\prime \prime}(\omega) \cos \omega t\right) \\
& \times\left(\delta_{i k}\left(n_{j} n_{l}\right)+\delta_{j k}\left(n_{i} n_{l}\right)-\frac{2}{3} \delta_{i j} n_{k} n_{l}\right) \\
& +\left(G_{3}^{\prime}(\omega) \sin \omega t+G_{3}^{\prime \prime}(\omega) \cos \omega t\right) \\
& \times\left(n_{i} n_{j}-\frac{1}{3} \delta_{i j}\right) n_{k} n_{l} \gamma_{0 k l} .
\end{aligned}
$$

We may write down each component explicitly.

$$
\begin{aligned}
\Sigma_{x x} & =\left(G_{1}^{\prime}(\omega)+\frac{4}{3} G_{2}^{\prime}(\omega)+\frac{2}{3} G_{3}^{\prime}(\omega)\right) \\
& \times \gamma_{0 x x} \sin \omega t \\
& +\left(G_{1}^{\prime \prime}(\omega)+\frac{4}{3} G_{2}^{\prime \prime}(\omega)+\frac{2}{3} G_{3}^{\prime \prime}(\omega)\right) \\
& \times \gamma_{0 x x} \cos \omega t \\
\Sigma_{y y} & =G_{1}^{\prime}(\omega) \gamma_{0 y y} \sin \omega t+G_{1}^{\prime \prime}(\omega) \gamma_{0 y y} \cos \omega t \\
& +\left(-\frac{2}{3} G_{2}^{\prime}(\omega)-\frac{1}{3} G_{3}^{\prime}(\omega)\right) \gamma_{0 x x} \sin \omega t \\
& +\left(-\frac{2}{3} G_{2}^{\prime \prime}(\omega)-\frac{1}{3} G_{3}^{\prime \prime}(\omega)\right) \gamma_{0 x x} \cos \omega t, \\
\Sigma_{x y} & \left(G_{1}^{\prime}(\omega)+G_{2}^{\prime}(\omega)\right) \frac{1}{2}\left(\gamma_{0 x y}+\gamma_{0 y x}\right) \sin \omega t \\
& +\left(G_{1}^{\prime \prime}(\omega)+G_{2}^{\prime \prime}(\omega)\right) \frac{1}{2}\left(\gamma_{0 x y}+\gamma_{0 y x}\right) \cos \omega t, \\
& =\left(G_{1}^{\prime}(\omega)\right) \frac{1}{2}\left(\gamma_{0 y z}+\gamma_{0 z y}\right) \sin \omega t \\
\Sigma_{y z} & \left(G_{1}^{\prime \prime}(\omega)\right) \frac{1}{2}\left(\gamma_{0 y z}+\gamma_{0 z y}\right) \cos \omega t,
\end{aligned}
$$

where only the traceless part is taken into consideration because the trace part corresponds to the pressure, and the total pressure is determined by the incompressibility condition. We may define similarly the functions $G_{i}^{r}(\omega)$ and $G_{i}^{d}(\omega)$, $(i=1,2,3)$ for the reversible parts and the dissipative part of the stress tensor, respectively.

In the case of an oriented lamellar structure, there are three fundamental configurations for applying shear flow as shown in Fig. 1. In the perpendicular configuration, the normal of lamellae is perpendicular to both the flow direction and the velocity gradient direction. In the parallel configuration, the normal is parallel to the velocity gradient direction but perpendicular to the flow direction. In the transverse configuration, the normal is parallel to the flow direction but perpendicular to the velocity gradient direction. It is readily shown that when the lamellar structure is periodic along the $\mathrm{x}$ axis, the stress in the flow direction for the parallel and the transverse configuration is given by $\Sigma_{x y}=\Sigma_{y x}$ whereas the stress for the perpendicular configuration is given by $\Sigma_{y z}$.

It is also remarked that three kinds of complex moduli in eqn (29) is consistent with the fact that there are three viscoelastic constants for lamellar structures under the incompressibility condition ${ }^{39}$.

\section{Explicit form of the stress tensor}

Now we discuss the response of the stress tensor. In order to evaluate the reversible part of the stress tensor given by eqn (25), we solve eqn (22) and (23) by the perturbation expansion 


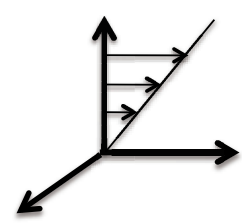

Shear Flow

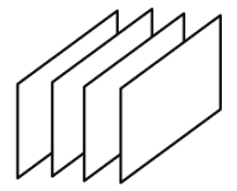

Transverse

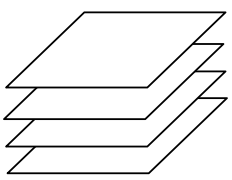

Parallel

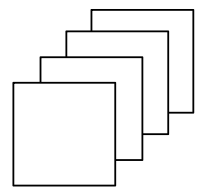

Perpendicular

Fig. 1 Parallel, perpendicular and transverse configurations of lamellae under shear flow.

in terms of the strain $\gamma_{i j}$ and the coupling constant $\beta$. In the weak segregation limit, the concentration profile and the orientational order parameter profile can be written, respectively, as

$$
\begin{gathered}
\phi(\vec{r}, t)=\bar{\phi}+\left(A(t) e^{i \vec{q} \cdot \vec{r}}+c . c .\right), \\
Q_{i j}(\vec{r}, t)=\bar{Q}_{i j}(t)+\left(B_{i j}(t) e^{2 i \vec{q} \cdot \vec{r}}+c . c .\right),
\end{gathered}
$$

where $\vec{q}$ is the fundamental reciprocal lattice vector for aligned lamellae, which may depend on time as eqn (76) in Appendix $B$ under time-dependent strain. Without loss of generality, we may assume that the amplitude $A$ is real. It is readily understood that the period of $Q_{i j}$ is half of that of $\phi$. In order to avoid complication, we assume that the elastic coefficient $C$ for the orientational order parameter is sufficiently large so that the spatial variation of $Q_{i j}$ can be ignored, i.e., $B_{i j}=0$. Substituting (34) and $Q_{i j}(\vec{r}, t)=\bar{Q}_{i j}(t)$ into eqn (22) and (23), or eqn (80) and (81) in Appendix C, one can obtain the solutions in powers of $\gamma$ and $\beta$.

As the details of the calculation are shown in Appendix B, and Appendix $\mathrm{C}$, we obtain the traceless part of the reversible stress tensor in terms of $A^{(1)}, \bar{Q}_{i j}^{(1)}$, and $\gamma_{i j}(t)$ up to $\mathrm{O}(\gamma)$ and $\mathrm{O}(\beta)$ as

$$
\Sigma_{i j}^{r}=\Sigma_{e l, i j}^{r}+\Sigma_{i j}^{r(1,0)}+\beta \Sigma_{i j}^{r(1,1)},
$$

where

$$
\begin{aligned}
\Sigma_{e l, i j}^{r} & =8 \alpha \gamma_{x x} A^{(0) 2} \frac{1}{q^{4}}\left(q_{i} q_{j}-\frac{1}{3} \delta_{i j} q^{2}\right) \\
\Sigma_{i j}^{r(1,0)} & =\lambda a \bar{Q}_{i j}^{(1,0)} \\
\Sigma_{i j}^{r(1,1)} & =2\left[Q_{i x}^{(1,0)} \delta_{j x}+Q_{j x}^{(1,0)} \delta_{i x}-\frac{2}{3} Q_{x x}^{(1,0)} \delta_{i j}\right] q^{2} A^{(0) 2} \\
& +\lambda\left[a \bar{Q}_{i j}^{(1,1)}\right. \\
& \left.+2\left(\delta_{i x} \gamma_{x j}(t)+\delta_{j x} \gamma_{x i}(t)-\frac{2}{3} \delta_{i j} \gamma_{x x}(t)\right) q^{2} A^{(0) 2}\right] .
\end{aligned}
$$

The expressions of $A^{(0)}, Q_{i j}^{(1,0)}$ and $Q_{i j}^{(1,1)}$ are given in Appendix C. The term $\Sigma_{e l, i j}^{r}$ provides us with an elastic constant of lamellar structure. The terms proportional to $\gamma_{\alpha \beta}(t)=$ $\gamma_{0 \alpha \beta} \sin \omega t$ describe the elastic response whereas those proportional to $\cos \omega t$ the dissipative response.

From the results for $\bar{Q}_{i j}$, we obtain each component as

$$
\begin{aligned}
\Sigma_{x y}^{r(1,0)}= & \frac{a \lambda^{2}}{2}\left(\gamma_{0 x y}+\gamma_{0 y x}\right) M(\omega) \\
& \times\left(\cos \omega t+\frac{\omega}{\Gamma a} \sin \omega t\right), \\
\Sigma_{x y}^{r(1,1)} & =\frac{\lambda}{2}\left(\gamma_{0 x y}+\gamma_{0 y x}\right) 4 q^{2} A^{(0) 2} M(\omega) \\
& \times\left(\frac{\omega}{\Gamma a} \sin \omega t+\cos \omega t\right) . \\
\Sigma_{e l, x x}^{r} & =\frac{16}{3} \gamma_{0 x x} \alpha q^{-4} A^{(0) 2} \sin \omega t, \\
\Sigma_{x x}^{r(1,0)}= & a \lambda^{2} \gamma_{0 x x} M(\omega)\left(\cos \omega t+\frac{\omega}{\Gamma a} \sin \omega t\right), \\
\Sigma_{x x}^{r(1,1)}= & \lambda \gamma_{0 x x} \frac{16}{3} q^{2} A^{(0) 2} M(\omega) \\
\times & \left(\frac{\omega}{\Gamma a} \sin \omega t+\cos \omega t\right) . \\
\Sigma_{e l, y y}^{r}= & -\frac{8}{3} \gamma_{0 x x} \alpha q^{-4}\left|A^{(0)}\right|^{2} \sin \omega t, \\
\Sigma_{y y}^{r(1,0)}= & a \lambda^{2} \gamma_{0 y y} M(\omega)\left(\cos \omega t+\frac{\omega}{\Gamma a} \sin \omega t\right), \\
\Sigma_{y y}^{r(1,1)}= & -\lambda \gamma_{0 x x} \frac{8}{3} q^{2} A^{(0) 2} M(\omega) \\
\times & \left(\frac{\omega}{\Gamma a} \sin \omega t+\cos \omega t\right) . \\
\Sigma_{y z}^{r(1,0)}= & \frac{a \lambda^{2}}{2}\left(\gamma_{0 y z}+\gamma_{0 z y}\right) M(\omega) \\
\times & \left(\cos \omega t+\frac{\omega}{\Gamma a} \sin \omega t\right) .
\end{aligned}
$$

where

$$
M(\omega)=\frac{\omega /(\Gamma a)}{1+(\omega /(\Gamma a))^{2}}
$$


We have dropped some terms by noting that $q^{2}=\sqrt{\alpha / L}+$ $O\left(\beta^{2}\right)$ as eqn (88) in Appendix C. It is noted that only the symmetric part of the velocity gradient contributes to the viscoelastic response, because the antisymmetric part represents the uniform rotation of the system. Eqn (42) for $\Sigma_{e l, x x}^{r}$ gives us the elastic response due to the deformation of lamellar structures, which exists even when the coupling between the orientational order parameter and the concentration field is absent. $\Sigma_{i j}^{r(1,0)}$ represents the viscoelastic response of molecular alignment due to the flow and this contribution exists even when there is no microphase separated structure. The viscoelastic response $\Sigma_{i j}^{r(1,1)}$ which is proportional to $A^{(0) 2}$ arises from the microphase separated structures and the coupling between the orientational order parameter and the concentration field. The characteristic relaxation rate relevant to $\Sigma_{i j}^{r(1,0)}$ and $\Sigma_{i j}^{r(1,1)}$ is $\Gamma a$, which is the relaxation frequency of the orientational order parameter. If we take the Rouse dynamics for individual chains, this relaxation rate is proportional to $N^{-2}$ with $N$ the molecular weight ${ }^{35}$. When $\omega$ is much larger (smaller) than $\Gamma a$, these responses reduce to the elastic (viscous) response. This situation would change if we take into account the second order contribution of the coupling $\beta$, because it may contain the relaxation rate of composition fluctuations.

Finally the above results for the macroscopic reversible stress tensor can be rewritten in the form of eqn (29) by setting

$$
\begin{gathered}
G_{1}^{\prime r}(\omega)=a \lambda^{2} \frac{\omega}{\Gamma a} M(\omega), \\
G_{1}^{\prime \prime}(\omega)=a \lambda^{2} M(\omega), \\
G_{2}^{\prime r}(\omega)=4 \lambda \beta q^{2}\left|A^{(0)}\right|^{2} \frac{\omega}{\Gamma a} M(\omega), \\
G_{2}^{\prime \prime r}(\omega)=4 \lambda \beta q^{2}\left|A^{(0)}\right|^{2} M(\omega),
\end{gathered}
$$

and

$$
\begin{aligned}
& G_{3}^{\prime r}(\omega)=8 \alpha q^{-4}\left|A^{(0)}\right|^{2}, \\
& G_{3}^{\prime \prime}(\omega)=0 .
\end{aligned}
$$

Therefore, we obtain a Maxwell-type complex moduli $G_{1}^{r}$ and $G_{2}^{r}$ up to the linear order of the coupling constant $\beta$. We note that in the limit $\omega \rightarrow 0$, only the storage modulus $G_{3}^{\prime}(\omega)$ remains finite. Yamada and Ohta ${ }^{40}$ calculated the elastic constants of several microphase separated structures. The elastic response given by (54) agrees with that for lamellar structure obtained by them. There are generally two kinds of elastic constants for lamellar structure or smectic A liquid crystals ${ }^{41}$. One is the dilation and the other is the bending of the layer structure. In our theory we have obtained only the dilation of the layer structure since the bending energy is represented by the higher spatial derivative.
Similarly, the macroscopic dissipative stress tensor can be derived from eqn (26) and the expressions of $\bar{Q}_{i j}$ in Appendix C. The final results are given by

$$
\begin{array}{r}
G_{1}^{\prime d}(\omega)=G_{2}^{\prime d}(\omega)=G_{3}^{\prime d}(\omega)=0, \\
G_{1}^{\prime \prime d}(\omega)=\left(2 v_{1}-\frac{4}{3} S v_{2}\right) \omega, \\
G_{2}^{\prime \prime d}(\omega)=2 v_{2} S \omega, \\
G_{3}^{\prime \prime} d(\omega)=0 .
\end{array}
$$

where $S=2(\beta / a) q^{2} A^{(0) 2}$.

\section{Concluding remarks and discussion}

In this paper, we have formulated a linear vsicoelastic theory for aligned lamellar structures in microphase separated diblock copolymers. We have applied the mode expansion method in the weak segregation limit, and evaluated the linear viscoelastic response up to the first order of the coupling constant $\beta$ between the composition gradient and the molecular orientation.

We can evaluate the viscosities of lamellar structure from the theory developed here. It is well known that there are three kinds of viscosities for incompressible fluids with uniaxial symmetry ${ }^{17,20}$. The macroscopic dissipative stress tensor can be written as

$$
\begin{aligned}
\Sigma_{i j}^{D}= & \alpha_{1} n_{i} n_{j} n_{k} n_{l} A_{k l}+\alpha_{4} A_{i j}+\alpha_{5} n_{k} n_{i} A_{k j}+\alpha_{6} n_{k} n_{j} A_{k i} \\
& -\frac{1}{3} \delta_{i j}\left(\alpha_{1}+\alpha_{5}+\alpha_{6}\right) n_{k} n_{l} A_{k l},
\end{aligned}
$$

with $\alpha_{5}=\alpha_{6}$. From eqn (58) and (59), and taking the $\omega \rightarrow 0$ limit of the loss moduli (51) and (53), we obtain

$$
\alpha_{1}=0
$$

$$
\begin{gathered}
\alpha_{4}=2\left(v_{1}-\frac{2}{3} S v_{2}\right)+\frac{\lambda^{2}}{\Gamma}, \\
\alpha_{5}=\alpha_{6}=2 v_{2} S+2 \frac{\lambda S}{\Gamma} .
\end{gathered}
$$

In the disordered state at high temperature, we may put $S=0$ so that $\alpha_{5}=\alpha_{6}=0$ and $\alpha_{4}=2 v_{1}$. Comparing this with eqn (62), one notes that the viscosity $\alpha_{4}$ for the perpendicular configuration decreases in the lamellar phase provided that the coefficient $v_{2}$ which appears in eqn (21) is positive. Since the loss modulus for the parallel configuration in the low frequency limit is given by $\alpha_{4}+\alpha_{5}$, this is larger than that for the perpendicular configuration since the coefficient $\lambda$ in eqn (23) is positive as we have assumed. 
As long as we have noticed, the complex moduli (50)(59) and the viscosities (61)-(63) are new results. There have been no theories to formulate these linear viscoelastic quantities for lamellar structures in diblock copolymer melts. There are some rheological experiments for aligned lamellar block copolymers. Koppi et $\mathrm{al}^{42}$ carried out dynamical mechanical measurements for the three lamellar configurations shown in Fig. 1 in sheared diblock copolymer melts. At higher frequency, the dynamical elastic moduli as well as the frequency-dependent viscosities are not distinguishable for the three configurations whereas these take different values for smaller frequencies. For example, the viscosity for perpendicular lamellae is smaller than that of parallel lamellae consistent qualitatively with our results mentioned above. However, their data for the perpendicular configuration become different from those for the transverse configuration. Theoretically the shear stress for these two configurations should be equal to each other due to the symmetry $\Sigma_{x y}=\Sigma_{y x}$. This discrepancy at low frequency may be originated from some nonlinear and/or nonequilibirium effects. Shear alignment and rheological measurements have also been conducted for lamellar Polystyrene-Polyisoprene block copolymers by Patel et al ${ }^{43}$ where the loss modulus for parallel lamellae near the orderdisorder transition temperature is larger than that of perpendicular ones in the double logarithmic plot as a function of frequency scaled by the shift factor. This trend is again consistent with our results. A quantitative comparison seems impossible because of the large contrast in the viscoelastic properties between the styrene and isoprene blocks in the experiments while our theory assumes dynamical symmetry for the two blocks. We note that there is another experiment for lamellar Polystyrene-Polyisoprene block copolymers ${ }^{44}$ in which the loss modulus of parallel configuration is smaller than that of perpendicular configuration. However, the experiment was carried out at $120^{\circ} \mathrm{C}$ which is not close to the order-disorder transition $164{ }^{\circ} \mathrm{C}$ and the data are outside applicability of the present theory in the weak segregation. Hahn et al ${ }^{38}$ have carried out experiments of lamellar block copolymers minimizing defect density. Their data for the best aligned sample of perpendicular configuration indicate that $G^{\prime} \propto \omega^{2}$ and $G^{\prime \prime} \propto \omega$ for the low frequency regime consistent with the present results.

The present study implies that one has to choose carefully the relevant dynamical variables for microphase separated structures. As mentioned in the Introduction, the equilibrium phase diagram has been obtained successfully by mean field theories essentially in terms of local concentrations ${ }^{1,6-8}$. The static elastic theory has also been formulated based on the similar theories ${ }^{40,45}$. Dynamics of phase ordering and morphological transitions have been studied extensively by the time-evolution equations for the local concentrations $22,46-48$. However, the viscoelastic properties cannot be obtained properly only by the local concentrations. This can be seen from the reversible part of the macroscopic stress tensor (25) which is given in the absence of the variable $Q_{i j}$ by

$$
\frac{1}{V} \int d \vec{x} \sigma_{i j}^{r}=-\sum_{\vec{q}} q_{i} q_{j}\left[L-\frac{\alpha}{q^{4}}\right] \phi_{\vec{q}} \phi_{-\vec{q}},
$$

where $\phi_{\vec{q}}$ is the Fourier component of $\phi(\vec{r})$ with $\vec{q}$ the fundamental reciprocal vectors. The strain dependence of $q_{i}$ as (76) in Appendix B gives us the elastic constants. The dissipation due to deformation arises from the relaxation of the concentration $\phi_{\vec{q}}$. It is obvious that, by expanding $\phi_{\vec{q}}$ in terms of the shear rate, the stress (64) can produce a term in the form of the $\alpha_{1}$ term in eqn (60) provided that $\vec{q}$ is proportional to $\vec{n}$. However, it is impossible to obtain other terms in eqn (60). In the present study, we have considered the orientational degrees of freedom and have shown that they contribute to the dissipative part of the viscoelastic response in lamellar structure.

\section{Acknowledgements}

One of the authors (T.O.) would like to thank Dr. A. Menzel for valuable discussions at the early stage of this study. This work was supported by the JSPS Core-to-Core Program "International research network for non-equilibrium dynamics of soft matter" and the Grant-in-Aid for the Global COE Program "The Next Generation of Physics, Spun from Universality and Emergence" from the Ministry of Education, Culture, Sports, Science and Technology (MEXT) of Japan. One of the authors (S. Y.) acknowledges support from JSPS (Grant No. 241799), and the other (T. O.) from JSPS (Grand No. 23540449 and 24244063).

\section{A Derivation of the reversible macroscopic stress tensor}

We derive the macroscopic stress tensor $\Sigma_{i j}^{r}$ by calculating the free energy deviation by the following infinitesimal uniform deformation $\vec{u}(\vec{x})$

$$
\begin{gathered}
\vec{r}=\vec{x}+\vec{u}(\vec{x}), \\
u_{i}(\vec{x})=\left(\nabla_{j} u_{i}\right) x_{j} .
\end{gathered}
$$

Here we assume that $\left(\nabla_{j} u_{i}\right)$ is constant in space. Throughout this paper, we will use the coordinate $r_{i}$ for the moving frame, and $x_{i}$ for the laboratory frame. 
The variables $\phi$ and $Q_{i j}$ are transformed as

$$
\begin{aligned}
\phi^{\prime}(\vec{r})= & \phi(\vec{x}) \\
Q_{i j}^{\prime}(\vec{r})= & Q_{i j}(\vec{x})+\frac{1}{2} Q_{i k}\left(\nabla_{k} u_{j}-\nabla_{j} u_{k}\right) \\
& +\frac{1}{2} Q_{j k}\left(\nabla_{k} u_{i}-\nabla_{i} u_{k}\right) \\
& +\frac{1}{2} \lambda_{i j k l}\left(\nabla_{k} u_{l}+\nabla_{l} u_{k}\right) \\
= & Q_{i j}(\vec{x})+\left(Q_{i k} W_{k j}+Q_{j k} W_{k i}\right)+\lambda_{i j k l} C_{k l}
\end{aligned}
$$

Here we have defined the symmetric deviation gradient tensor $C_{i j}$ and the antisymmetric deviation gradient tensor $W_{i j}$ as

$$
\begin{aligned}
C_{i j} & =\frac{1}{2}\left(\nabla_{i} u_{j}+\nabla_{j} u_{i}\right), \\
W_{i j} & =\frac{1}{2}\left(\nabla_{i} u_{j}-\nabla_{j} u_{i}\right) .
\end{aligned}
$$

The gradient term is transformed by

$$
\nabla_{i}=\nabla_{i}^{\prime}+\left(\nabla_{i} u_{j}\right) \nabla_{j}^{\prime}
$$

Here the prime symbols indicate the derivative in the coordinate system $\vec{r}$. The free energy deviation in the first order of $\vec{u}$ is calculated as follows.

$$
\begin{aligned}
\delta F & =\int d \vec{r} f\left(\left\{\phi^{\prime}(\vec{r}), Q_{i j}^{\prime}(\vec{r})\right\}\right) \\
& -\int d \vec{x} f\left(\left\{\phi(\vec{x}), Q_{i j}(\vec{x})\right\}\right) \\
& =2 \beta \int d \vec{x} Q_{i k}\left(\nabla_{k} \phi(\vec{x})\right)\left(\nabla_{j} \phi(\vec{x})\right) \nabla_{i} u_{j}(\vec{x}) \\
& +\int d \vec{x}\left(\psi_{k j} Q_{k i} \nabla_{i} u_{j}-\psi_{k i} Q_{k j} \nabla_{i} u_{j}\right) \\
+ & \int d \vec{x} \psi_{k l} \lambda_{k l i j} \nabla_{i} u_{j} \\
& -L \int d \vec{x}\left(\nabla_{j} \phi(\vec{x})\right)\left(\nabla_{i} \phi(\vec{x})\right) \nabla_{i} u_{j}(\vec{x}) \\
& -C \int d \vec{x}\left(\nabla_{j} Q_{k l}(\vec{x})\right)\left(\nabla_{i} Q_{k l}(\vec{x})\right) \nabla_{i} u_{j}(\vec{x}) \\
+ & \frac{\alpha}{2} \int d \vec{x} \int d \vec{y} \\
& {\left[\left(\nabla_{i} H(\vec{x}-\vec{y})\right) u_{i}(x)-\left(\nabla_{i} H(\vec{x}-\vec{y})\right) u_{i}(\vec{y})\right] } \\
\times & \phi(\vec{x}) \phi(\vec{y}) .
\end{aligned}
$$

In this derivation, we have employed eqn (67) and (68), and the following relations under the incompressibility condition

$$
\begin{aligned}
& -L \int d \vec{x}\left(\nabla_{i} \nabla_{j} \phi(\vec{x})\right)\left(\nabla_{i} \phi(\vec{x})\right) u_{j}(\vec{x}) \\
& =\frac{L}{2} \int d \vec{x}\left(\nabla_{i} \phi(\vec{x})\right)\left(\nabla_{i} \phi(\vec{x})\right) \nabla_{j} u_{j}(\vec{x})=0,
\end{aligned}
$$

$$
\begin{aligned}
& 2 \beta \int d \vec{x} Q_{i j}\left(\nabla_{j} \phi(\vec{x})\right)\left(\nabla_{i} \nabla_{k} \phi(\vec{x})\right) u_{k}(\vec{x}) \\
& +\beta \int d \vec{x}\left(\nabla_{k} Q_{i j}\right)\left(\nabla_{j} \phi(\vec{x})\right)\left(\nabla_{i} \phi(\vec{x})\right) u_{k}(\vec{x}) \\
= & -\beta \int d \vec{x} Q_{i j}\left(\nabla_{j} \phi(\vec{x})\right)\left(\nabla_{i} \phi(\vec{x})\right) \nabla_{k} u_{k}(\vec{x})=0 .(74)
\end{aligned}
$$

By substituting the expression of $\psi_{i j}$ given by eqn (16), we can further simplify this expression as

$$
\begin{aligned}
\delta F= & \beta \int d \vec{x}\left[Q_{i k}\left(\nabla_{k} \phi(\vec{x})\right)\left(\nabla_{j} \phi(\vec{x})\right)\right. \\
+ & \left.Q_{j k}\left(\nabla_{k} \phi(\vec{x})\right)\left(\nabla_{i} \phi(\vec{x})\right)\right] \nabla_{i} u_{j} \\
+ & \int d \vec{x} \psi_{k l} \lambda_{k l i j} \nabla_{i} u_{j} \\
& -\quad L \int d \vec{x}\left(\nabla_{j} \phi(\vec{x})\right)\left(\nabla_{i} \phi(\vec{x})\right) \nabla_{i} u_{j} \\
- & C \int d \vec{x}\left(\nabla_{j} Q_{k l}(\vec{x})\right)\left(\nabla_{i} Q_{k l}(\vec{x})\right) \nabla_{i} u_{j} \\
+ & \frac{\alpha}{2} \int d \vec{x} \int d \vec{y} \\
& {\left[\left(\nabla_{j} H(\vec{x}-\vec{y})\right)\left(x_{i}-y_{i}\right) \nabla_{i} u_{j}\right] \phi(\vec{x}) \phi(\vec{y}) . }
\end{aligned}
$$

From this, we may define the stress tensor as $\int d \vec{x} \sigma_{i j}^{r} \nabla_{i} u_{j}$ and obtain the formula given by eqn (25).

\section{B Calculation of the viscoelastic response}

In this Appendix, we describe the details of calculation of the viscoelastic response. First we express the reversible stress tensor in terms of the amplitude $A$ and $\bar{Q}_{i j}$ up to the first order of the strain $\gamma$. We define the time dependent wave number as

$$
q_{i}(t)=q_{0 i}-\gamma_{j i}(t) q_{0 j}
$$

where $\vec{q}_{0}$ is the fundamental reciprocal lattice vector of undeformed lamellae. Substituting eqn (34), (35), and (82) in Appendix C into eqn (25), we obtain

$$
\begin{aligned}
& \Sigma_{i j}^{r}=\left[-2 L q_{i}(t) q_{j}(t)+2 \alpha \frac{q_{i}(t) q_{j}(t)}{q(t)^{4}}\right] A^{(0) 2} \\
+ & 2 \beta q_{k}(t)\left[\bar{Q}_{i k}^{(1)}(t) q_{j}(t)+\bar{Q}_{j k}^{(1)}(t) q_{i}(t)\right] A^{(0) 2} \\
+ & \lambda\left[a \bar{Q}_{i j}^{(1)}\right. \\
+ & 2 \beta\left(q_{i} \gamma_{k j}(t) q_{k}+q_{j} \gamma_{k i}(t) q_{k}-\frac{2}{3} \delta_{i j} q_{k} \gamma_{l k}(t) q_{l}\right) A^{(0) 2} \\
- & \left.4 \beta\left(q_{i} q_{j}-\frac{1}{3} \delta_{i j} q^{2}\right)\left(A^{(0)} A^{(1)}\right)\right]+O\left(\gamma^{2}, \beta^{2}\right) .
\end{aligned}
$$


Using eqn (76), this can be evaluated as

$$
\begin{aligned}
& \Sigma_{i j}^{r}=2\left(L-\frac{\alpha}{q^{4}}\right) A^{(0) 2}\left(\delta_{i x} \gamma_{x j}(t)+\delta_{j x} \gamma_{x i}(t)\right) \\
+ & 8 \alpha \gamma_{x x} \frac{q_{i} q_{j}}{q^{4}} A^{(0) 2}+2 \beta\left[\bar{Q}_{i x}^{(1)} \delta_{j x}+\bar{Q}_{j x}^{(1)} \delta_{i x}\right] q^{2} A^{(0) 2} \\
+ & \lambda\left[a \bar{Q}_{i j}^{(1)}\right. \\
+ & 2 \beta\left(\delta_{i x} \gamma_{x j}(t)+\delta_{j x} \gamma_{x i}(t)-\frac{2}{3} \delta_{i j} \gamma_{x x}(t)\right) q^{2} A^{(0) 2} \\
- & \left.4 \beta\left(\delta_{i x} \delta_{j x}-\frac{1}{3} \delta_{i j}\right) q^{2}\left(A^{(0)} A^{(1)}\right)\right] \\
+ & O\left(\gamma^{2}, \beta^{2}\right) .
\end{aligned}
$$

The first term on the right hand side vanishes because of the equilibrium condition $q^{2}=\sqrt{\alpha / L}$ as eqn (88).

\section{Perturbation expansions}

Here we obtain the solutions of the time-evolution equations calculated by the perturbation expansion. We introduce the new coordinate $\vec{r}$ which moves with the flow

$$
r_{i}(t)=x_{i}-\gamma_{i j}(t) x_{j}
$$

In the moving frame, the time evolution equations are given as

$$
\begin{aligned}
\frac{\partial \phi}{\partial t}= & D \hat{\nabla}^{2}\left[-L \hat{\nabla}^{2} \phi-\theta \phi+g \phi^{3}+2 \beta \hat{\nabla}_{i}\left(Q_{i j} \hat{\nabla}_{j} \phi\right)\right] \\
- & D \alpha(\phi-\bar{\phi}) \\
\frac{\partial Q_{i j}}{\partial t} & +\quad Q_{i k} \Omega_{k j}+Q_{j k} \Omega_{k i}-\lambda_{i j k l} A_{k l} \\
& =-\Gamma\left[a Q_{i j}-\beta\left(\hat{\nabla}_{i} \phi\right)\left(\hat{\nabla}_{j} \phi\right)+\frac{\beta}{3}\left(\hat{\nabla}_{i} \phi\right)^{2} \delta_{i j}\right] \\
& +C \hat{\nabla}^{2} Q_{i j},
\end{aligned}
$$

where

$$
\hat{\nabla}_{i} \equiv \nabla_{i}-\gamma_{j i} \nabla_{j}
$$

The operator $\nabla_{i}$ contained in $\Omega_{k i}$ and $A_{k l}$ should not be replaced by $\hat{\nabla}_{i}$ since it acts on the local velocity. See Appendix A.

In order to solve eqn (80) and (81) in the weak segregation limit, we put $\phi(\vec{r}, t)$ and $Q_{i j}(\vec{r}, t)$ as eqn (34) and (35), respectively and make an expansion of the amplitudes in powers of the strain $\gamma$;

$$
\begin{aligned}
A & =A^{(0)}+A^{(1)}+O\left(\gamma^{2}\right), \\
\bar{Q}_{i j} & =\bar{Q}_{i j}^{(0)}+\bar{Q}_{i j}^{(1)}+O\left(\gamma^{2}\right) .
\end{aligned}
$$

The zeroth order solutions are given as follows. From eqn (81) we have

$$
a \bar{Q}_{i j}^{(0)}(\vec{r})-\beta\left(\nabla_{i} \phi^{(0)}(\vec{r}) \nabla_{j} \phi^{(0)}(\vec{r})-\frac{1}{3}\left|\vec{\nabla} \phi^{(0)}\right|^{2} \delta_{i j}\right)=0 .
$$

By substituting eqn (83), the amplitude $\bar{Q}_{i j}^{(0)}$ is given by

$$
\bar{Q}_{i j}^{(0)}=\frac{2 \beta}{a} q^{2}\left|A^{(0)}\right|^{2}\left(\delta_{i x} \delta_{j x}-\frac{1}{3} \delta_{i j}\right) .
$$

From eqn (80) and (83), we obtain

$$
\begin{aligned}
& -q^{2}\left(L q^{2} A^{(0)}-\theta A^{(0)}+3 g A^{(0)}\left|A^{(0)}\right|^{2}+3 g \bar{\phi}^{2} A^{(0)}\right) \\
& -\alpha A^{(0)}=0,
\end{aligned}
$$

where $O\left(\beta^{2}\right)$ terms are omitted. The magnitude of the wave number $q$ is determined from the minimization of the free energy. By substituting eqn (86) and (83) into eqn (2), we obtain

$$
\frac{\partial F}{\partial q}=L-\alpha q^{-4}+O\left(\beta^{2}\right)=0
$$

and hence $q^{2}=\sqrt{\alpha / L}$.

At the first order of the shear strength $\gamma$, equations for the amplitude $A^{(1)}$ and $\bar{Q}_{i j}^{(1)}$ are given, respectively, by

$$
\begin{aligned}
\frac{d A^{(1)}}{d t} & =-3 g D q^{2}\left|A^{(0)}\right|^{2} A^{(1)}+2 D \beta q^{4} A^{(0)} \bar{Q}_{x x}^{(1)} \\
& +O\left(\beta^{2}\right) \\
\frac{d \bar{Q}_{x x}^{(1)}}{d t} & =-\Gamma a \bar{Q}_{x x}^{(1)}+\frac{8}{3} \Gamma \beta q^{2} A^{(0)} A^{(1)}+\lambda \gamma_{0 x x} \omega \cos \omega t \\
& -\frac{8}{3} \Gamma \beta q^{2}\left|A^{(0)}\right|^{2} \gamma_{0 x x} \sin \omega t \\
& +\frac{4}{3} \Gamma \beta q^{2}\left|A^{(0)}\right|^{2} \gamma_{0 x x} \sin \omega t \\
\frac{d \bar{Q}_{y y}^{(1)}}{d t} & =-\Gamma a \bar{Q}_{y y}^{(1)}-\frac{4}{3} \Gamma \beta q^{2} A^{(0)} A^{(1)}+\lambda \gamma_{0 y y} \omega \cos \omega t \\
\frac{d \bar{Q}_{x y}^{(1)}}{d t} & =-\Gamma a \bar{Q}_{x y}^{(1)} \\
& -\beta q^{2}\left|A^{(0)}\right|^{2}\left(\Gamma \sin \omega t+\frac{\omega}{a} \cos \omega t\right)\left(\gamma_{0 x y}-\gamma_{0 y x}\right) \\
& +\left(\frac{1}{2} \lambda \omega \cos \omega t-\Gamma \beta \sin \omega t q^{2}\left|A^{(0)}\right|^{2}\right) \\
& \times\left(\gamma_{0 x y}+\gamma_{0 y x}\right) \\
\frac{d \bar{Q}_{y z}^{(1)}}{d t} & =-\Gamma a \bar{Q}_{y z}^{(1)}+\frac{1}{2} \lambda\left(\gamma_{0 y z}+\gamma_{0 z y}\right) \omega \cos \omega t
\end{aligned}
$$

We can solve these linear equations exactly, but the solution is complicated. Instead we expand these equations in terms of 
the coupling strength $\beta$ as

$$
\begin{aligned}
& \bar{Q}_{i j}^{(1)}=\bar{Q}_{i j}^{(1,0)}+\beta \bar{Q}_{i j}^{(1,1)}+O\left(\gamma \beta^{2}\right), \\
& A^{(1)}=A^{(1,0)}+\beta A^{(1,1)}+O\left(\gamma \beta^{2}\right) .
\end{aligned}
$$

The lowest order contributions $\bar{Q}_{x x}^{(1,0)}$ and $A^{(1,0)}$ satisfy

$$
\begin{aligned}
\frac{d}{d t}\left(\begin{array}{c}
\bar{Q}_{x x}^{(1,0)} \\
A^{(1,0)}
\end{array}\right) & =\left(\begin{array}{cc}
-\Gamma a & 0 \\
0 & -\Gamma_{A}
\end{array}\right)\left(\begin{array}{c}
\bar{Q}_{x x}^{(1,0)} \\
A^{(1,0)}
\end{array}\right) \\
& +\left(\begin{array}{c}
\lambda \omega \cos \omega t \\
0
\end{array}\right) \gamma_{0 x x},
\end{aligned}
$$

where

$$
\Gamma_{A}=3 g D q^{2} A^{(0) 2}
$$

The asymptotic solutions for $t \rightarrow \infty$ are given by $A^{(1,0)}=0$ and

$$
\bar{Q}_{x x}^{(1,0)}=\frac{\lambda \omega}{\Gamma a} N(\omega)\left(\cos \omega t+\frac{\omega}{\Gamma a} \sin \omega t\right) \gamma_{0 x x},
$$

where

$$
N(\omega)=\frac{1}{1+(\omega / \Gamma a)^{2}} .
$$

Similarly we obtain

$$
\begin{aligned}
\bar{Q}_{x x}^{(1,1)} & =-\frac{8}{3 a} \gamma_{0 x x} q^{2} A^{(0) 2} N(\omega) \\
& \times\left(\sin \omega t-\frac{\omega}{\Gamma a} \cos \omega t\right) \\
\bar{Q}_{y y}^{(1,0)} & =\frac{\lambda \omega}{\Gamma a} \gamma_{0 y y} N(\omega) \\
& \times\left(\cos \omega t+\frac{\omega}{\Gamma a} \sin \omega t\right) \\
\bar{Q}_{y y}^{(1,1)} & =\frac{4}{3 a} q^{2} A^{(0) 2} \gamma_{0 x x} N(\omega) \\
\times & \left(\sin \omega t-\frac{\omega}{\Gamma a} \cos \omega t\right) \\
\bar{Q}_{x y}^{(1,0)} & \frac{\omega}{\Gamma a} \frac{\lambda}{2}\left(\gamma_{0 x y}+\gamma_{0 y x}\right) N(\omega) \\
\times & \left(\cos \omega t+\frac{\omega}{\Gamma a} \sin \omega t\right) \\
\bar{Q}_{x y}^{(1,1)} & -\left(\gamma_{0 x y}+\gamma_{0 y x}\right) \frac{q^{2}}{a} A^{(0) 2} N(\omega) \\
\times & \left(\sin \omega t-\frac{\omega}{\Gamma a} \cos \omega t\right) \\
& -\left(\gamma_{0 x y}-\gamma_{0 y x}\right) \frac{q^{2}}{a} A^{(0) 2} \sin \omega t \\
\bar{Q}_{y z}^{(1)} & =\frac{\omega}{\Gamma a} \frac{\lambda}{2}\left(\gamma_{0 y z}+\gamma_{0 z y}\right) N(\omega) \\
& \left(\cos \omega t+\frac{\omega}{\Gamma a} \sin \omega t\right) \\
& =
\end{aligned}
$$

It is noted that, since $A^{(1,0)}=0$, there are no contributions to $\bar{Q}_{i j}^{(1,0)}$ and $\bar{Q}_{i j}^{(1,1)}$ from $A^{(1)}$, and that $\bar{Q}_{y z}^{(1)}$ has no contribution from the spatial variation of the concentration as can be seen from eqn. (93).

\section{References}

1 M. W. Matsen and M. Schick, Phys. Rev. Lett., 1994, 72, 2660.

2 S. Forster, A. K. Khandpur, J. Zhao, F. S. Bates, I. W. Hamley, A. J. Ryan and W. Bras, Macromolecules, 1994, 27, 6922 .

3 A. K. Khandpur, S. Foerster, F. S. Bates, I. W. Hamley, A. J. Ryan, W. Bras, K. Almdal and K. Mortensen, Macromolecules, 1995, 28, 8796.

4 M. W. Matsen and F. S. Bates, Macromolecules, 1996, 29, 1091.

5 M. W. Matsen and F. S. Bates, Journal of Chemical Physics, 1997, 106, 2436.

6 E. W. Cochran, C. J. Garcia-Cervera and G. H. Fredrickson, Macromolecules, 2006, 39, 2449.

7 C. A. Tyler and D. C. Morse, Phys. Rev. Lett., 2005, 94, 208302.

8 K. Yamada, M. Nonomura and T. Ohta, Journal of Physics-Condensed Matter, 2006, 18, L421.

9 A. Ranjan and D. C. Morse, Phys. Rev. E, 2006, 74, 011803.

10 M. Takenaka, T. Wakada, S. Akasaka, S. Nishitsuji, K. Saijo, H. Shimizu, M. I. Kim and H. Hasegawa, Macromolecules, 2007, 40, 4399.

11 B. Miao and R. A. Wickham, J. Chem. Phys., 2008, 128, 054902.

12 M. B. Kossuth, D. C. Morse and F. S. Bates, J. Rheol., 1999, 43, 167.

13 M. E. Vigild, K. Almdal, K. Mortensen, I. W. Hamley, J. P. A. Fairclough and A. J. Ryan, Macromolecules, 1998, 31, 5702.

14 C.-Y. Wang and T. P. Lodge, Macromolecules, 2002, 35, 6997.

15 G. Floudas, R. Ulrich and U. Wiesner, J. Chem. Phys., 1999, 110, 652.

16 M. F. Schulz, F. S. Bates, K. Almdal and K. Mortensen, Phys. Rev. Lett., 1994, 73, 86.

17 C. D. Yoo and J. Vinals, Macromolecules, 2012, 45, 4848.

18 G. Giupponi, J. Harting and P. V. Coveney, Europhys. Lett., 2006, 73, 533.

19 R. Tamate, K. Yamada, J. Vinals, and T. Ohta, J. Phys. Soc. Jpn., 2008, 77, 034802.

20 J. L. Ericksen, J. Arch. Rational Mech. Anal., 1959, 4, 231. 21 Y. Matsushita, K. Mori, Y. Mogi, R. Saguchi, I. Noda, M. 
Nagasawa, T. Chang, C. Glinka and C. C. Han, Macromolecules 1990, 23, 4317.

22 K. Yamada, M. Nonomura and T. Ohta, Macromolecules, 2004, 37, 5762.

23 I. W. Stewart and F. Stewart, J. Phys.: Condens. Matter, 2009, 21, 465101.

24 D. R. M. Williams and F. C. MacKintosh, Macromolecules, 1994, 27, 7677.

25 G. Fredrickson, J. Rheol., 1994, 38, 1045.

26 F. Drolet, P. Chen, and J. Vinals, Macromolecules, 1999, 32, 8603.

27 G. K. Auernhammer, H. R. Brand and H. Pleiner, Rheologica Acta, 2000, 39, 215.

28 G. K. Auernhammer, H. R. Brand and H. Pleiner, Phys. Rev. E, 2002, 66, 061707.

29 A. S. Wunenburger, A. Colin, T. Colin and D. Roux, Eur. Phys. J. E, 2000, 2, 277.

30 T. Ohta and K. Kawasaki Macromolecules, 1986, 19, 2621.

31 P. G. de Gennes, Scaling concepts in polymer physics, Cornell University Press, New York 1979.

32 M. Doi, J. Phys. Condens. Matter, 2011, 23, 284118.

33 H. Pleiner, M. Liu, and H.R. Brand, IMA Proceedings in Mathematics and its Applications, Springer, New York, 2005, 141, 99.

34 K. Kawasaki and T. Ohta, Physica A, 1983, 118, 175

35 M. Doi and S. F. Edwards, The Theory of Polymer Dynamics, Oxford University Press, London, 1986.

36 K. Kawasaki and A. Onuki, Phys. Rev. A, 1990, 42, R3664.

37 M. Rubinstein and S. P. Obukhov, Macromolecules, 2001, 348701

38 H. Hahn, J. H. Lee, N. P. Balsara, B. A. Garetz and H. Watanabe, Macromolecules, 1993, 261740.

39 P. C. Martin, and O. Parodi, and P. S. Pershan, Phys. Rev. A, 1972, 6, 2401.

40 K. Yamada and T. Ohta, Europhys. Lett., 2006, 73, 614.

41 P. G. de Gennes and J. Prost, The Physics of Liquid Crystals, Clarendon Press, Oxford, 1993, 2nd ed.

42 K. A. Koppi, M. Tirrell, F. S. Bates, K. Almdal and R. H. Colby, J. Phys. II France, 1992, 2, 1941.

43 S. S. Patel, R. G. Larson, K. I. Winey and H. Watanabe, Macromolecules, 1995, 284313.

44 V. K. Gupta, R. Krishnamoorti, J. A. Kornfield and S. D. Smith, Macromolecules, 1996, 291359.

45 C. A. Tyler and D. C. Morse, Macromolecules, 2003, 36 8184.

46 M. Bahiana and Y. Oono, Phys. Rev. A, 1990, 41, 6763.

47 T. Kawakatsu, Statistical Physics of Polymers, SpringerVerlag, Heidelberg, 2004.

48 J. G. E. M. Fraaije, J. Chem. Phys., 1993, 99, 9202. 